\title{
Alimentación de dos poblaciones de perca sol (Lepomis gibbosus), introducidas en el Norte de España. Resultados preliminares
}

\author{
J. Domínguez', J.C. Pena', J. De Soto² y E. Luis ${ }^{2}$ \\ 'Departamento de Zoología. Universidad de León. Campus de Vegazana. 24071 León. España. \\ *Departamento de Ecología, Genética y Microbiología. Área de Ecología. Universidad de León. Campus de \\ Vegazana. 24071 León. España.
}

\section{RESUMEN}

Se estudia la alimentación de la perca sol en el Norte de España y se compara la dieta de dos poblaciones, Embalse de Albina (Alava) y en las lagunas de las graveras de Bustillo (Palencia), donde ha sido introducida en fechas recientes.

Se han analizado los contenidos estomacales de 70 ejemplares del embalse y 141 de las graveras capturados en primavera. La tasa de ingesta, medida como porcentaje de estómagos con contenido es elevada en ambos casos, como es usual en poblaciones de reciente introducción, y varía entre el $86 \%$ en Albina y $\mathbf{7 6} \%$ en Bustillo.

El espectro de la dieta es semejante en ambas poblaciones. En el embalse el número de taxones presa es de 17 y en las graveras de 15. El número de taxones preferenciales es reducido en los dos casos y difieren en las poblaciones estudiadas, mientras en el embalse de Albina la perca sol se alimenta, preferentemente, de Quironómidos y Cladóceros, en las graveras de Bustillo lo hace sobre Copépodos y Cladóceros. Estas diferencias se relacionan con la naturaleza de las masas de agua y con la densidad de las poblaciones estudiadas.

Palabras clave: perca sol, alimentación.

\begin{abstract}
The diet of the pumpkinseed (Lepomis gibbosus) in Northern Spain was studied. Two populations were compared, that of the Albina reservoir (Alava, Spain) and that of the Bustillo gravel pit (Palencia, Spain), where it has been recently introduced. We analysed the stomach contents of 70 specimens from the reservoir and 141 from the gravel pit captured during Spring. The ingestion rate (i.e. thepercentage of individuals with a full stomach), was high. This is a common observation in recently introduced populations.

The diet composition was similar in both populations. In the reservoir, the number of different items was 17, while it was 15 in the gravel pit. In the Albina reservoir, the pumpkinseed fed mostly on Chironomidae and Cadocera, while in theBustillo ponds theyfed on Copepoda and Cladocera. The differences in diet composition between sites are related to both environmental features and to pumpkinseed population densities.
\end{abstract}

Keywords: pumpkinseed, diet composition

\section{INTRODUCCIÓN}

En los últimos años se ha puesto de relieve, en no pocas ocasiones, el progresivo deterioro que vienen sufriendo las poblaciones de peces fluviales en nuestro país. La introducción de especies forá- neas es uno de los factores más importantes que inciden en el estado de conservación de la fauna autóctona (Rincón et al., 1990;Elvira, 1995 a y b, 1998)). Las perturbaciones producidas en las comunidades ícticas preestablecidas hacen preciso el estudio de la biología de especies alóctonas 
que permita orientar la gestión tendente a minimizar sus efectos.

Por ello el objetivo del presente trabajo es contribuir al conocimiento de la alimentación de la perca sol (Lepomis gibbosus) en dos masas de agua, lo que permitirá determinar la incidencia que puedan ejercer sobre el resto de especies de la comunidad. Las poblaciones estudiadas proceden una del embalse de Albina en Álava y otra de las graveras de Bustillo en Palencia. En ambos casos la aparición de la perca sol es reciente, en torno a 1994, sin que pueda precisarse con exactitud, pues se trata de introducciones fraudulentas que van dispersando esta especie por toda la Península Ibérica, como recogen Sostoa et al. (1987), Asensio \& Pinedo (1993) y Asensio et al. (1996).

\section{ÁREA DE ESTUDIO}

El embalse de Albina se localiza en la comarca del Gorbea, en el sector septentrional del territorio histórico de Álava, cuyo paisaje presenta crestones calizos y cuestas de forma sucesiva. $\mathrm{Su}$ finalidad es la captación de agua para consumo y su principal tributario es el arroyo de Albina. El llenado del embalse se inició en 1945 por lo que, en la actualidad, está en fase de madurez. Climatológicamente se encuentra en el tercio Sur del dominio climático oceánico. Desde el punto de vista geológico predominan las estructuras del Cretácico inferior con materiales blandos de margas estructuradas en capas con estratos muy finos en los que se alternan calizas, areniscas y cuarcitas con margas y arcillas. El embalse dispone de escasa vegetación sumergida y carece de orla de vegetación acuática.

Por su parte las graveras de Bustillo de la Vega se encuentran ubicadas en la parte central de la provincia de Palencia, área denominada de fondo de valle y llanuras aluviales siguiendo la clasificación del Mapa geológico de Castilla y León. Desde este punto de vista presentan materiales pelítico-arcillosos con abundantes cantos, como corresponde a depósitos de origen fluvial cuyo origen data del Terciario y forman parte del relleno de la cuenca del río Carrión. Son el resultado del abandono de una explotación de áridos en los depósitos aluviales clausurada en 1994. Como consecuencia se ha producido un sistema de ocho lagunas someras, cuya profundidad máxima oscila entre 1 y $2 \mathrm{~m}$, intercomunicadas entre si.

El agua de llenado procede, fundamentalmente, de infiltración del nivel freático, con aporte de dos arroyos periféricos. Los valores de los parámetros físico-químicos del agua analizados $(\mathrm{pH}$, conductividad, alcalinidad, sólidos en suspensión, DBO, DQO, amonio total, nitratos, fósforo y sulfatos) son los correspondientes a un tramo de río salmonícola, con excepción de la $\mathrm{DBO}$, en cuyo caso se corresponde en tres de los puntos de muestreo con aguas salmonícolas y en otros once estaciones de muestreo con las características de aguas ciprinícolas. No se detectaron niveles valorables de los metales pesados analizados, $\mathrm{Zn}, \mathrm{Cd}$ y $\mathrm{Pb}$. Por el contrario resultaron elevados los valores de sólidos en suspensión en todas las estaciones de muestreo así como los niveles de mineralización, detectados a través de los valores de conductividad.

La vegetación sumergida se limita a Ceratophyllum demersum y el contorno de las lagunas presenta una orla de Typha latifolia, Juncus glaucus e Iris pseudacorus.

\section{MATERIAL Y MÉTODOS}

Para la evaluación de la comunidad íctica del embalse de Albina se utilizaron técnicas de ecointegración combinadas con métodos de muestreo directo para conocer la composición de la comunidad, utilizando en este caso una red de arrastre de $60 \mathrm{~m}$ de longitud, $2.5 \mathrm{~m}$ de altura y paso de malla de $16 \mathrm{~mm}$. La sonda empleada ha sido una FURUNO FE 4300 con transductor SOB-NR a una frecuencia de $50 \mathrm{khz}$ con un ángulo de barrido de $46^{\circ}$ que emite ondas cada 0.2 milisegundos. Se realizaron un total de 12 transectos en la primavera de 1998 rastreando bandas de profundidades 0-10 m y 10-20 m. El cálculo de la densidad se ha establecido basándose en las capturas por métodos directos y por conteos de ecos. 
Si bien los ecos no permiten valorar los ejemplares de tallas inferiores a $18 \mathrm{~cm}$, lo que excluiría de esta estima de densidad a la perca sol, especie dominante en este embalse. Por ello se recurrió a efectuar una segunda estima de la densidad a partir de las capturas por arrastre mediante la red de barrido, utilizando para ello la expresión de Daget y Pettit (1971).

En las lagunas de Bustillo para efectuar la estima de los efectivos se ha recurrido a la disposición de artes pasivas: copos y nasas. Las dimensiones de los primeros fueron boca de $2.5 \times 3 \mathrm{~m}$, con tres cámaras y un copo final de $6 \mathrm{~m}$; los paños laterales son de $15 \times 2.5 \mathrm{~m}$ y malla de luz de $16 \mathrm{~mm}$. Por su parte las nasas dispuestas tenían un diametro horizontal de $65 \mathrm{~cm}$ y altura de 52 $\mathrm{cm}$; constan de 5 cámaras con trampa que finalizan en un cono de $240 \mathrm{~cm}$ de longitud. La luz de malla es de $80 \mathrm{~mm}$. Se dispusieron 3 series de 4 nasas cada una que se mantuvieron durante día y medio cada una durante una semana, durante la primavera de 1999.

La estima de los efectivos se llevó a cabo, en este caso, por el método del área restringida (Granado, 1996).

De los ejemplares capturados se midió y peso una parte alícuota representativa, lo que permitió calcular, posteriormente, la biomasa. Además algunos individuos de perca sol fueron conservados, en formol, al $8 \%$ para realizar el estudio de la dieta; lo fueron 70 del embalse de Albina y 147 de las lagunas de Bustillo.

Se estimó el coeficiente de alometría (b) de la relación longitud-peso $\mathrm{Ps}=\mathrm{a} \mathrm{LT}^{\mathrm{b}}$ y el índice de condición o coeficiente de Fulton, a partir de la expresión $\mathrm{K}=100 \mathrm{Ps} / \mathrm{LT}^{3}$.

Las presas contenidas en los estómagos fueron determinadas y se estimó, para cada grupo, la frecuencia de aparición sobre el total de estómagos que contenían algún alimento y la proporción numérica, abundancia, en que se encontraron representadas. Para realizar el análisis gráfico de la dieta se han seguido las propuestas de Costello (1990) y Tokechi (1991). El primero de ellos utiliza la representación conjunta de los dos descriptores de la dieta, frecuencia de aparición y abundancia de cada uno de los taxones presa. Por
Tabla 1. Composición de la comunidad ictica en las dos zonas de estudio. Especies presentes y proporción de perca sol. La densidad máxima estimada corresponde al total de la comunidad. Composition of the fish community in the two study areas. Fish species present and percentage of the total community mude up $0 j$ pumpkinseed. The maximum density estimated is reluted to the community total.

\begin{tabular}{|c|c|c|}
\hline $\begin{array}{l}\text { Composición de la } \\
\text { comunidad íctica }\end{array}$ & $\begin{array}{c}\text { Embalse de } \\
\text { Albina }\end{array}$ & $\begin{array}{l}\text { Lagunas de } \\
\text { Bustillo }\end{array}$ \\
\hline \multicolumn{3}{|l|}{ Trucha común } \\
\hline Salmo trutta & $\mathrm{X}$ & \\
\hline \multicolumn{3}{|l|}{ Trucha arco iris } \\
\hline Oncorhynchus mykiss & & $\mathrm{X}$ \\
\hline \multicolumn{3}{|l|}{ Lucio } \\
\hline Esox lucius & $\mathrm{X}$ & \\
\hline \multicolumn{3}{|l|}{ Barbo } \\
\hline Barbus bocagei & & $\mathrm{X}$ \\
\hline \multicolumn{3}{|l|}{ Loina } \\
\hline Chondrostoma tturiense & $\mathrm{X}$ & \\
\hline \multicolumn{3}{|l|}{ Escallo } \\
\hline Squalius carolitertii & & $\mathrm{X}$ \\
\hline \multicolumn{3}{|l|}{ Tenca } \\
\hline Tinca tinca & $\mathrm{X}$ & $\mathrm{X}$ \\
\hline \multicolumn{3}{|l|}{ Bermejuela } \\
\hline Chondrostoma arcasii & & $X$ \\
\hline \multicolumn{3}{|l|}{ Gobio } \\
\hline Gobio gobio & & $X$ \\
\hline \multicolumn{3}{|l|}{ Black-bass } \\
\hline Micropterus salmoides & $\mathrm{X}$ & \\
\hline \multicolumn{3}{|l|}{ Perca-coi } \\
\hline Lepomis gibbosus & $94.29 \%$ & $90.14 \%$ \\
\hline
\end{tabular}

Densidad máxima estimada $220 \mathrm{ind}$./ha $142.305 \mathrm{ind} . / \mathrm{ha}$

su parte el método propuesto por Tokeshi representa la diversidad total de la población y la media de las diversidades de los ejemplares analizados. La afinidad entre las dietas de las dos masas de agua estudiadas fue valorada cualitativamente mediante el índice de Sorensen (1948) y cuantitativamente mediante el de Motyka et al. (1950). Además para conocer el solapamiento de las dietas se calculó el índice simplificado de Morisita (Horn, 1966).

\section{RESULTADOS}

En las dos masas de agua estudiadas la perca sol resultó ser la especie numéricamente dominante. 


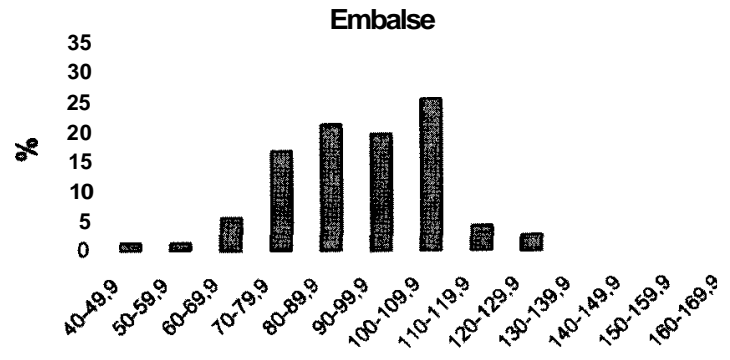

LT (mm)

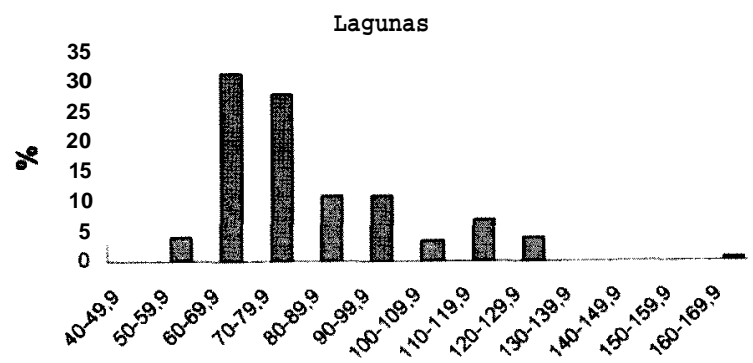

LT (mm)

Figura 1. Distribución de longitudes de los ejemplares objeto de estudio (LT: longitud total; \%: proporción de cada clase de talla). Length-frequency distribution of the specimens studied (LT: total length, \%: proportion of individuals in euch size class)

En el embalse de Albina la densidad estimada fue de 220 ejemplares por Ha. En las lagunas de Bustillo la densidad obtenida alcanzó valores muy elevados, 142.305 ejemplares/Ha. La biomasa estimada fue en el caso del embalse de 699 $\mathrm{kg} / \mathrm{Ha}$ y $1704 \mathrm{~kg} / \mathrm{Ha}$ en las lagunas estudiadas. La composición cualitativa de las comunidades ícticas establecidas en cada caso, así como la proporcion representada de perca sol y las densidades estimadas se muestran en la tablai. En ambos casos la perca sol representa más del $90 \%$ de los individuos de las comunidades ícticas respectivas. Por otra parte cabe destacar que en los dos casos la presencia de especies exóticas es elevada en las dos comunidades, particularmente en el caso del embalse.

La longitud total media de los ejemplares de perca sol capturados en el embalse de Albina fue de $89.17 \mathrm{~mm}$, con una desviación estándar de $15.8 \mathrm{~mm}$; el ejemplar más pequeño medía $40 \mathrm{~mm}$
Embalse de Albina

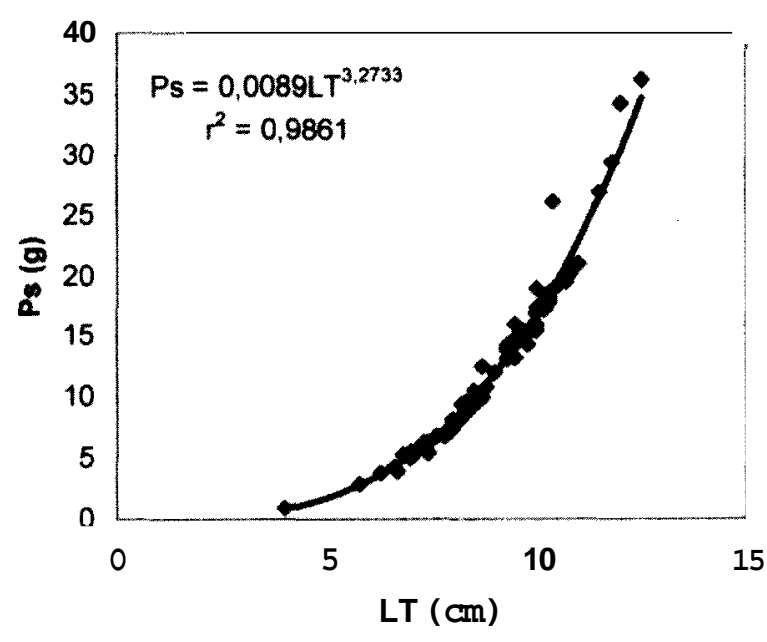

Lagunas de Bustillo

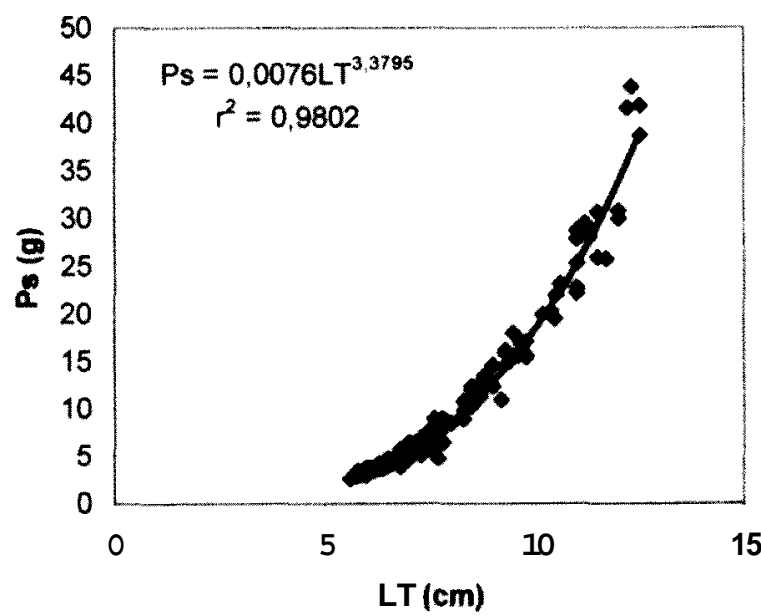

Figura 2. Relación longitud / peso de cada una de las poblaciones estudiadas. (LT longitud total; Ps: peso; r: coeficiente de correlación. Length-weight relationship of each of the studied populations (LT: total length, Ps: weight, r: correlation coefficient).

y el de mayor talla alcanzó los $125 \mathrm{~mm}$.. Por su parte aquellos procedentes de las graveras de Bustillo poseían una longitud total media de $79.80 \mathrm{~mm}$, con desviación estándar de $18.96 \mathrm{~mm}$; las tallas mínima y máxima registradas fueron, respectivamente, de 56 y $165 \mathrm{~mm}$. La distribu- 
Tabla 2. Presas consumidas por Lepomis gibbosus en ambas localidades de estudio. (\%F: frecuencia de aparición; \%N: proporción numérica). Preys consumed by the pumpkinseed at sampling sites (\% F frequency of occurrence; $\% N$ : numerical rate).

\begin{tabular}{|c|c|c|c|c|}
\hline & \multicolumn{2}{|c|}{ Embalse de Albina } & \multicolumn{2}{|c|}{ Lagunas de Bustillo } \\
\hline & $\% \mathrm{~F}$ & $\% \mathrm{~N}$ & $\% \mathrm{~F}$ & $\% \mathrm{~N}$ \\
\hline Cladocera & 71.67 & 48.87 & 59.46 & 30.30 \\
\hline Copepoda & & & 62.16 & 29.34 \\
\hline Glossiphonidae & & & 0.90 & 0.02 \\
\hline Chironomidae & 45.00 & 41.03 & 14.41 & 0.51 \\
\hline Ceratopogonidae & 1.67 & 0.15 & & \\
\hline Tipulidae & & & 0.90 & 0.02 \\
\hline Cordulegasteridae & & & 0.90 & 0.02 \\
\hline Gomphidae & 1.67 & 0.15 & 1.80 & 0.04 \\
\hline Corixidae & 3.33 & 0.45 & & \\
\hline Osmylidae & 1.67 & 0.15 & & \\
\hline Coleoptera (imagos) & & & 0.90 & 0.02 \\
\hline Ephemeridae & 5.00 & 1.06 & 0.90 & 0.02 \\
\hline Baetidae & 3.33 & 0.60 & 2.70 & 0.24 \\
\hline Perlidae & 3.33 & 0.30 & & \\
\hline Capniidae & 6.67 & 0.75 & & \\
\hline Limnephilidae & 3.33 & 0.45 & & \\
\hline Odontoceridae & 6.67 & 1.06 & & \\
\hline Rhyacophilidae & 1.67 & 0.15 & & \\
\hline Polycentropodidae & 3.33 & 0.45 & 1.80 & 0.04 \\
\hline Hydropsychidae & & & 0.90 & 0.02 \\
\hline Sialidae & 3.33 & 0.60 & & \\
\hline Huevos de peces & 6.67 & 3.77 & 17.12 & 24.33 \\
\hline Escamas & & & 34.23 & 15.07 \\
\hline Restos vegetales & 8.33 & & 2.70 & \\
\hline
\end{tabular}

ción de longitudes de los ejemplares objeto de estudio se representa en la figura 1.

La relación entre la longitud y el peso en cada una de las poblaciones toma la forma representada en la figura 2. Como puede observarse el coeficiente de alometría resultó más elevado, 3.38, en las Lagunas de Bustillo, frenta a 3.27 en el embalse de Albina, lo que sugiere que el peso se incrementa en las lagunas en mayor proporción que en el embalse a lo largo de la vida de los peces. Este extremo se ve confirmado por un índice de condición también más elevado en las lagunas que en el embalse, 1.68 y 1.62 respectivamente.

Por lo que se refiere a la tasa de ingesta, medida como proporción de estómagos con contenido, ha resultado elevada, $86 \%$ en Albina y $76 \%$ en Bustillo. La amplitud del espectro de la dieta también resulto semejante en cuanto a la riqueza de items presentes. En el embalse de Albina el número de grupos de presas consumido fue de 17 y en las graveras de Bustillo de 15. Sin embargo sí existen diferencias en cuanto a los taxones de los que se alimentan, como puede observarse en la Tabla 2. Cualitativamente la afinidad mostrada por el índice de Sorensen es del 50\%. Cuantitativamente la proximidad de la dieta ha sido medida por dos índices diferentes. Los resultados al aplicar el porcentaje de similitud de Motyka fueron $47.73 \%$ cuando se utiliza la frecuencia de los diferentes taxones presa y $34.92 \%$ cuando se utiliza la abundancia de los mismos. El índice de solapamiento de dieta de Morisita aplicado obtuvo valores de 0.61 y 0.48 respectivamente al emplear los parámetros referidos. Como puede 

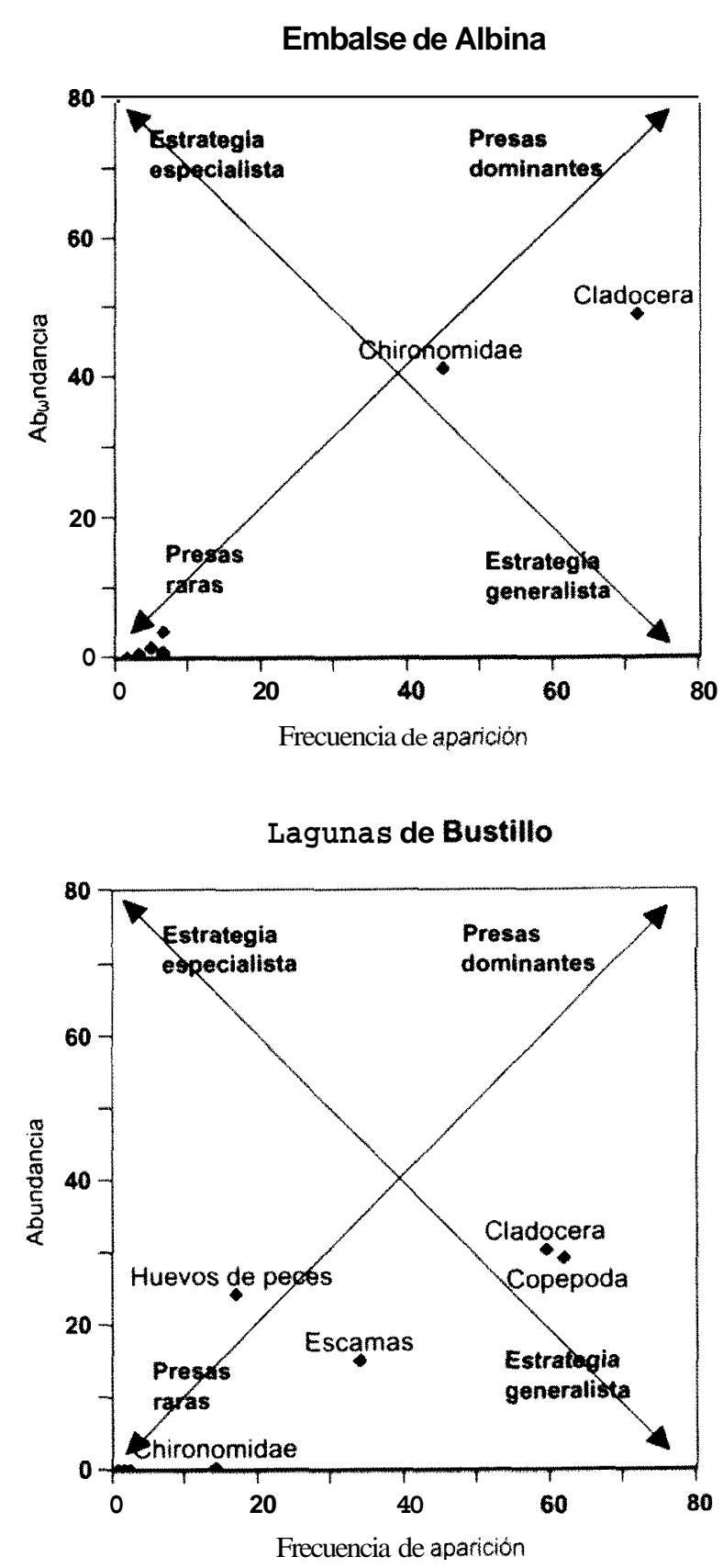

Figura 3. Análisis gráfico de la dieta de las poblaciones estudiadas (Costello, 1990). Graphical analysis of dietary data for the two populations studied (Costello, 1990). advertirse resultó más exigente para este fin el índice de Motyka.

Las diferencias en cuanto a la composición de la dieta reveladas por los índices aplicados se concretan, fundamentalmente, en los siguientes aspectos de la dieta. En Albina la perca sol consume preferentemente Cladóceros y larvas de Quironómidos, mientras en Bustillo ingiere Copépodos y Cladóceros como presas preferenciales. Además en este último punto hay que resaltar la depredación sobre huevos de peces y la ingesta de escamas de peces.

En la figura 3 se representan, siguiendo el método gráfico propuesto por Costello (1990), la frecuencia de aparición en abscisas y la abundancia de los diferentes grupos de presas en ordenadas, señalando los grupos mas relevantes. Siguiendo la interpretación sugerida por dicho autor la dieta en la población del embalse tiende hacia una especialización por los grupos señalados, mientras en las graveras la disposición de las presas sitúan a la población de perca sol en una fase claramente generalista de la dieta. Sin embargo cuando seguimos la interpretación propuesta por Tokeshi (1991), quien utiliza la representación conjunta de la diversidad de la población y la media de las diversidades individuales, las dos poblaciones se ubican claramente en el área de especies generalistas con alimentación heterogénea, según presenta en la figura 4 . Pese a ello la diversidad de la dieta resulta más elevada, como puede advertirse en el caso de la población correspondiente a las graveras.

\section{DISCUSIÓN}

La presencia de especies foráneas en las masas fluviales y la incidencias de éstas en las comunidades de especies nativas ha sido puesta de evidencia en no pocas ocasiones en la Península Ibérica. En nuestro pais este hecho ha sido evidenciado, entre otros, por Elvira (1995 a y b, 1998). Por su parte Godinho et al. (1997) refieren la presencia de 10 especies exóticas y 14 autóctonas en un estudio realizado sobre 35 embalses portugueses. Los resultados del presente estudio 
evidencian una vez más este hecho. En las dos masas de agua estudiadas es mayoritaria la presencia de especies alóctonas. Además en este caso numéricamente la perca sol representa en las dos ocasiones más del $90 \%$ de los efectivos totales, lo que revela la capacidad invasora de la especie. La presencia numerosa e incluso la sobrepoblación de perca sol ha sido referida previamente en nuestro pais por García de Jalón et al. (1993).

El rango de longitud de los ejemplares capturados resultó más amplio en las lagunas de Bustillo que en el Embalse de Albina. La ausencia de ejemplares más pequeños puede estar influenciada por el periodo de captura, previo a la reproducción de esta especie, lo que ha impedido la captura de ejemplares juveniles. No son distantes de los datos aportados por García de Jalón et al. (1993), quienes obtienen tallas comprendidas entre 30 y $160 \mathrm{~mm}$ (longitud ahorquillada), en varios embalses de la Comunidad de Madrid. Por su parte Brabrand et al. (1986) y Brabrand \& Salveit (1989), en dos embalses portugueses, obtienen rangos de longitud muy estrechos, entre 98 y $130 \mathrm{~mm}$. Más extenso resulto el rango de longitudes en los ejemplares del lago de Bañolas objeto del estudio de García-Berthou \& Moreno-Amich (2000) quienes muestran tallas comprendidas entre 19 y 176 $\mathrm{mm}$ que, por otra parte efectuaron los muesteos estacionalmente a lo largo del año.

Por lo que se refiere a la relación longitud-peso en los ejemplares capturados el coeficiente de alometría resultó más elevado en las poblaciones estudiadas que aquellas referidas por García de Jalón et al. (1993), lo que revela mayor incremento en peso que en las poblaciones de referencia. El índice de condición estimado en las dos poblaciones estudiadas se aproxima al aportado por por García de Jalón et al. (1993) en el embalse de Pedrezuela (1.63), donde refieren una sobrepoblación de esta especie. Sin embargo son más coincidentes con los obtenidos en el embalse de Albina que en las lagunas de Bustillo, pese a ser mucho más elevada la abundancia estimada en estas últimas. Los valores de este mismo índice presentados por Fox \& Keast (1990) también se aproximan a nuestros resultados. Estos autores

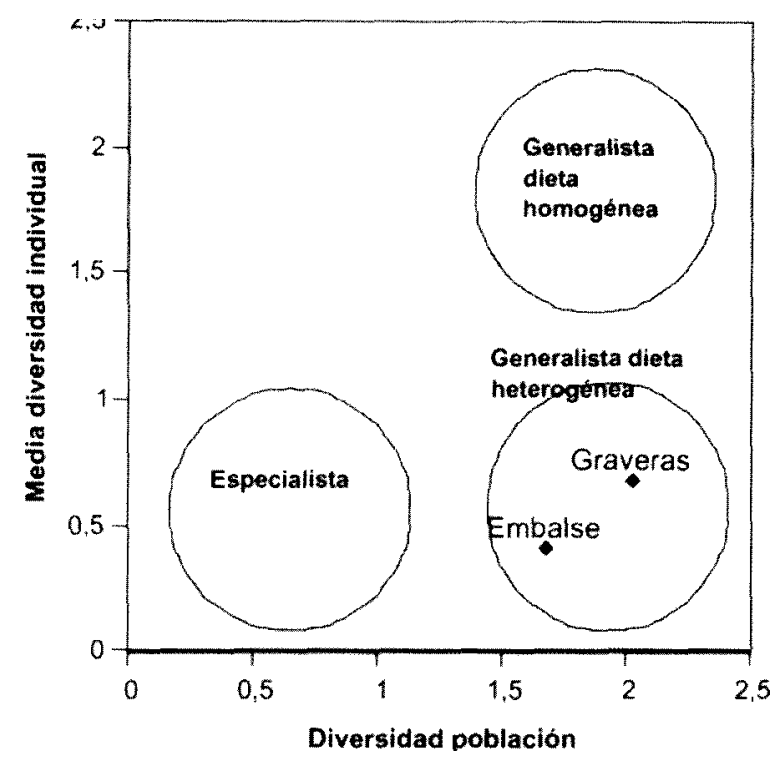

Figura 4. Representación del análisis gráfico propuesto por Tokeshi (1991). Graphical analysis as proposed by Tokeshi (1991).

obtienen valores próximos a 1.9 en primavera, que descienden hacia el verano en los estanques estudiados, por el contrario en el lago estudiado los valores próximos a 1.6 en primavera que aumenta hasta coincidir en el verano con los estimados en los estanques.

Centrándonos en el estudio de la dieta, en primer lugar cabe señalar que la ingesta resultó elevada en los dos casos, el valor más pequeño en las lagunas puede ser debido a la sobrepoblación que existe, como puede advertirse por los resultados de densidad estimada.

Si nos referimos a la riqueza de la dieta, entendida como número de grupos de presas contenidos en la dieta, si bien la disparidad de niveles taxonómicos referidos por los diferentes autores no son equivalentes, sí permiten dar una idea de la variabilidad de presas consumidas. Únicamente los datos aportados por García Berthou \& Moreno-Amich (2000), con 18 items (de ellos 4 son diferentes especies de crutáceos), superan los 17 y 15 respectivamente en el embalse y lagunas objeto del presente estudio. Brabrand et al. (1986) hallaron 10, 6 y 4 grupos de presas en los 
tres embalses portugueses de su estudio. Fox \& Keast (1990) describen hasta 7 grupos de presas en tres lagos canadienses. Por su parte Rodríguez Jimenez (1989) en un estudio realizado sobre perca sol en un embalse extremeño determina 12 items, mientras Godinho \& Ferreira (1998) describen 14 items en la dieta de esta especie en un río portugués de la cuenca del Tajo y atribuyen el elevado número de taxones presentes al oportunismo alimentario de esta especie, que ya había sido referido por Zapata y Granado Lorencio (1993) y García de Jalón et al. (1993) en sus respectivos estudios sobre diferentes embalses españoles. El elevado número de items consumido ha de atribuirse al oportunismo alimentario de esta especie señalado por Godinho et al. (1997).

Por lo que se refiere a la diferencia entre las presas consumidas las dos masas de agua estudiadas puede ser debida a la disponibilidad de presas en el medio; la interpretación de dichas diferencias exigiría un estudio sobre dicha disponibilidad, si bien Godinho et al. (1997) demuestran selectividad sobre determinadas presas. La mayor diversidad de la dieta en la población de perca sol de las lagunas probablemente se vea influenciada por una mayor diversidad de la comunidad de macroinvertebrados y zooplancton presente en el medio, dadas las condiciones de agua somera, vegetación abundante, que la aproximan a estanques de cultivo.

Crustáceos del zooplancton son frecuentes yío abundantes en la dieta de esta especie según reflejan los trabajos de diversos autores (Brabrand et al., 1986, Brabrand \& Salveit, 1989, García-Berthou \& Moreno-Amich, 2000), con excepción de lo revelado por Rodríguez Jimenez (1989) en un embalse extremeño, donde ni los ejemplares de talla reducida consumen estos grupos de presas. Destacar la elevada presencia de Cladoceros en el embalse de Albina, que persiste aún en los ejemplares de mayor talla, mientras los Cladóceros y Copépodos consumidos por la perca sol en las lagunas únicamente aparecen en tallas inferiores a los $100 \mathrm{~mm}$. Estos mismos grupos aparecen solamente en ejemplares de menos de $50 \mathrm{~mm}$ en la cuenca del Guadiana (Godinho et al., 1997) y su ingesta se reduce a medida que se incrementa la talla del depredador (Godinho \& Ferreira (1998) si bien este hecho también puede estar influenciado por la diferente disponibilidad de presas en los sistemas Ióticos y Iénticos.

Rodríguez Jiménez (1989) pone de manifiesto una elevada presencia de formas preimaginales de diferentes grupos de insectos, particularmente de dípteros, lo que también coincide con nuestros resultados. Por el contrario la ingesta de Heterópteros expuesta por Brabrand et al. (1986), Brabrand \& Salveit (1989), Rodríguez Jiménez (1989), Godinho et al. (1997), Godinho \& Ferreira (1998), solo aparece en el presente estudio en una pequeña proporción de los ejemplares del embalse.

La ingesta de huevos de peces por esta especie ha tenido diversas interpretaciones. Por una parte ha sido considerada responsable de disminuir la capacidad reproductiva de otras especies con las que comparten hábitat (García de Jalón et al., 1993; Keith \& Allardi, 1998), lo que provoca la rarificación de éstas. Al igual que ocurre con la introducción de otras especies del género Lepomis (Lemly, 1985). Por otra GarcíaBerthou \& Moreno-Amich (2000) señalan que se trata de predación intraespecífica. En el caso que nos ocupa el periodo de muestreo no es coincidente con el reproductor de perca sol y en las lagunas de Bustillo se comprobó que en el momento de los muestreos era la bermejuela quien estaba efectuando la freza.

Cabe destacar que la predación relevante sobre inoluscos señalada por Mittelbach (1984) y Fox \& Keast (1990) no ha sido detectada.

La presencia de restos vegetales y escamas de peces ha sido referida por autores como Godinho et al. (1997), si bien en menor proporción que la estimada en este trabajo, fundamentalmente la presencia de escamas de peces en las lagunas de Bustillo, que puede ser interpretada como accidental al consumir las puestas de otros peces, en este caso de la bermejuela. 


\section{BIBLIOGRAFÍA}

ARENDT, J. \& D. WILSON. 1997. Optimistic growth: competition and an ontogenetic niche-shift select for rapid growth in pumpkinseed sunfish (Lepomis gibbosus). Evolution, 51(6): 1946-1954 .

ASENSIO, R. y J. PINEDO. 1993. invasores con escamas. Sustrai, 30: 70-73.

ASENSIO, R., J. PINEDO, y F. MARKINA. 1996. Revisión biogeográfica de la fauna piscícola del territorio histórico de Álava. Est. Mus. Cienc. Nat. De Alava, 10-11 : 305-318.

BRABRAND, A., S. J. SALVEIT, M. J. BROGUEIRA $\&$ G. CABEÇADAS. 1986. Fish distribution and density investigated by quantitative echosounding. Some ecological aspects of the fish fauna in three Portuguese reservoirs. Laboratorium for ferskvannsokologi og innlandsfiske. Raport nr. 89: $61 \mathrm{pp.}$

BRABRAND, A. \& S. J. SALVEIT. 1989. Ecological aspects of the fish fauna in three Portuguese reservoirs. Arch Hydrobiol., 114 (4) : 575-589.

COSTELLO, M. J. 1990. Predator feeding strategy and prey importance: a new graphical analysis. $J$. Fish Biol., 36:261-263.

DAGET, J. \& P. PETTIT. 1971.L'Echantillonnage des peuplements de poissons marins. En "Problémes d'Ecologie. L'echantillonnage des peuplements animaux des milieux aquatiques. Lamotte, M. \& F. Bourliere (eds.). Masson et Cie. Paris. 269-291.

ELVIRA, B. 1995 a. El declive de los peces fluviales en España. Ecosistemas, 22 : 66-71.

ELVIRA, B. 1995 b. Native and exotic freshwater fishes in Spanish river basins. Freshwat. Biol., 33: 103-108.

ELVIRA, B. 1998. Impact of introduced fish on the native freshwater fish fauna of Spain. In: Stocking and introduction of fish. Cowx, I.G. (ed.): 187-190. Oxford.

FOX, M. G. \& A. KEAST. 1990. Effects of winterkill on population structure, body size, and prey consumption patterns of pumpkinseed in isolated beaver ponds. Can. J. Zool., 68 (12) : 2489-2498.

GARCIAA-BERTHOU, E. \& R. MORENO-AMICH. 2000. Food of introduced pumkinseed sunfish: ontogenetic diet shift and seasonal variation. J. Fish Zool., 57: 29-40.

GARCÍA DE JALÓN, M. MAYO RUSTARAZO, B. GALLEGO \& F. HERVELLA. 1993. Las comunidades piscícolas de los embalses de Madrid. Directrices para su gestión. Ecologia, 7: 467-485.
GODINHO, F., M. T. FERREIRA \& R. V. CORTES. 1997. The environmental basis of diet variation in pumpkinseed sunfish, Lepomis gibbosus, and largemouth bass Micropterus salmoides, along an Iberian river basin. Env. Biol. Fih., 50: 105-115.

GODINHO, F. \& M. T. FERREIRA. 1998. Spatial variation in diet composition of pumpkinseed suntish, Lepomis gibbosus, and largemouth bass, Micropterus salmoides, from a Portuguese stream. Folia Zool., 47 (3): 205-213.

GRANADO LORENCIO, C. 1996. Ecologia de peces. Secretariado de Publicaciones. Universidad de Sevilla. Utrera (Sevilla). 353 pp.

HORN, H. S. 1966. Measurement of "overlap" in comparative ecological studies. Am Natur, 100: 419-423.

KEITH, P. \& J. ALLARDI. 1998. The introduced freshwater fish of France: status, impacts and management. In :Stocking and Introduction of Fish . Cowx, 1. G. (ed.). Hull International Fisheries Institute. University of Hull. Hull. 153-166

LEMLY, D. 1985. Suppression of Native Fish Populations by Green Sunfish in First-Order Streams of Piedmont North Carolina. Transactions of the American Fisheries Society, 114:705-712.

MITTELBACH, G. G. 1984. Predation and resource partitioning in two sunfishes (Centrarchidae). Ecology, 65: 499-5 13.

MOTYKA, J., B. DOBRZANSKI \& S. ZAWADZKI. 1950. Wstepne badania nad lakami pludniowo wschodniej Lubelszczyzny. Ann. Univ. Mariae Curie-Sklodowska Sect. E: Agricultura, 5:367-447.

RINCON, P. A., J. C. VELASCO, N. GONZÁLEZ \& C. POLLO. 1990. Fish assemblages in small streams in western Spain. The influence of an introduced predator. Arch. Hydrobiol., 118 (1): 81-91.

RODRÍGUEZ JIMÉNEZ, A. J. 1989. Hábitos alimentícios de Micropterus salmoides (Pisces: Centrarchidae), Lepomis gibbosus (Pisces: Centrarchidae) y Gambusia affinis (Pisces: Poecilidae) en las orillas del embalse de Proserpina (Extremadura, España). Limnetica, 5: 13-20.

SORENSEN, T. (1948). A method of stablishing groups of equal amplitude in plant sociology based on similarity of species content and its application to analysis of the vegetation on Danish Commons. Biologiske Skrifter, 5 (4): 1-34.

SOSTOA, A., J. LOBÓN-CERVIÁ, V. FERNÁNDEZ-COLOMÉ \& F.J. SOSTOA. 1987. La distribución del pez-sol (Lepomis gibbosus L.) 
en la Península Ibérica. Doñana, Acta Vertebrata, 14: 123-125.

TOKESHI, M. 1991. Graphical analysis of predator feeding ctrategy and prey importance. Freshwater Forum. $1: 179-183$.
ZAPATA, S. C. \& C. GRANADO-LORENCIO. 1993. Age, growth and feeding of the exotic cpecies Lepomis gibbosus in a Spanish cooling reservoir. Arch. Hydrobiol./Suppl., 90(4): 561-573. 